\title{
Bill Fibers Extraction Method Based on Global Statistic Decision and Local Adaptive Segmentation
}

\author{
Ting Han ${ }^{1}$, Jianbin $\mathrm{Xie}^{2}$, Tong $\mathrm{Liu}^{2, \mathrm{a}}$ \\ ${ }^{1}$ ZHONGCHAO Enterprise CO, LTD., Beijing, 100052 \\ ${ }^{2}$ College of Electronic Science and Engineering, National University of Defense Technology, \\ Changsha, 410073 \\ aliutong1129@126.com
}

Keywords: image segmentation, OTSU, adaptive segmentation, fiber extraction

\begin{abstract}
In order to solve the problem of high classifying error in the process of bill fibers extraction, an improved method is proposed for bill fibers extraction based on global statistic decision and local adaptive segmentation. First, locates the suspicious fibers regions in the $\mathrm{H}, \mathrm{S}$ color space of the global bill image according to the statistics of the fibers color. Then, in the $\mathrm{V}$ space of the suspicious fibers regions a more adaptive segmentation method called OTSU is adopted to carry out the fine division of local regions to acquire the precise fibers targets. Finally, mathematical morphology is employed to filter the fibers and at the same time reduces classifying error. The experiments show the proposed method extracts fibers from bill images with fewer classifying error and better segmentation result.
\end{abstract}

\section{Introduction}

With the fast development of our national economy, bills are used more and more widely. Under this circumstance, bill defrauding crimes arise and tend to increase, which have caused heavy economic loss to our country [1]. While the bill authenticity identification based on their fibers' characteristics can effectively prevent these crimes, it is difficult to extract these characteristic fibers. In the bill images of ultraviolet spectrum, the fibers show red and blue color and this forms the basis of bill fibers extraction. However, besides the fibers targets, there are words, frame, water solubility, flower patterns and so on in the bill complicated background. They have a very wide color range while the fibers targets are small and only have a significant color range in the local regions. They do not have this character in the global regions of bill's images. So such classical adaptive image segmentation methods as OTSU [2], the Maximum Entropy [3], K-means Clustering [4], etc. cannot extract fibers targets effectively. The methods in literature [5] and [6] are more adaptive in the color-image segmentation, but not effective in segmenting puny fibers targets. In order to improve the fibers extraction, this paper proposes to adopt two levels of segmentation: first, in the $\mathrm{H}, \mathrm{S}$ color space of bill image, the fibers targets are coarsely segmented to roughly locate the suspicious fibers regions; then, in these regions, the more adaptive method called OTSU is adopted to carry out the fine division in the V space; finally, mathematical morphology [7] is used to filter the suspicious fibers to get the fibers targets and reduces classifying error.

\section{Fibers detection}

In bill images of ultraviolet spectrum, there appear the fibers with the color of red and blue as is shown in Fig.1(a). Fig.1(b) is the magnified display of the local regions of some fibers. Apparently, there is a significant difference between the color range of the fiber's local regions and that of the bill's background image. However, in the global image, the fiber's color range is not significant and overlaps with part of the background. In order to detect the fibers targets accurately, this article suggests first switching the images in RGB format to the HSV color space, then roughly locating the fibers in the components of $\mathrm{H}$ and $\mathrm{S}$ to get the candidate regions, and finally finely dividing the fibers in the component of $\mathrm{V}$ to get the segmentation result. 

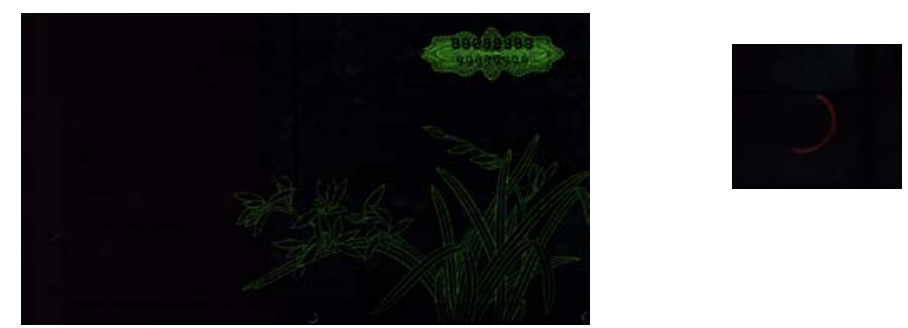

Fig.1 fibers in a bill image

(1) Color-space transformation

The images obtained via the image acquisition devices are in RGB format, but the component RGB is susceptible to the brightness of lighting source. Therefore it should be transformed to the color space of HSV. The equation of conversion is

$$
\left\{\begin{array}{l}
V=\max (R, G, B) \\
S=1-\frac{\min (R, G, B)}{V} \\
H=60 h
\end{array}\right.
$$

Where,

$$
\begin{aligned}
& 5+\frac{(V-B)}{\left[V-V_{\min }\right]} \quad, R=V \text { and } \quad G=V_{\text {min }} \\
& 1-\frac{(V-G)}{\left[V-V_{\min }\right]} \quad, R=V \quad \text { and } \quad B=V_{\text {min }} \\
& 1+\frac{(V-R)}{\left[V-V_{\min }\right]} \quad, G=V \quad \text { and } \quad B=V_{\min } \\
& 3-\frac{(V-B)}{\left[V-V_{\min }\right]} \quad, G=V \text { and } R=V_{\text {min }} \\
& 3+\frac{(V-G)}{\left[V-V_{\min }\right]} \quad, B=V \quad \text { and } \quad R=V_{\min } \\
& 5-\frac{(V-R)}{\left[V-V_{\min }\right]} \quad, B=V \quad \text { and } \quad G=V_{\text {min }} \\
& V_{\min }=\min (R, G, B)
\end{aligned}
$$

\section{(2) Rough location of Fibers}

The colors of bill fibers are red and blue. According to the statistics, their component $\mathrm{H}$ is in intervals [270, 360] and [160, 240], and their component S in interval [0.3, 0.6]. So, this article suggests adopting fixed threshold segmentation to roughly locate the fibers targets in the images' global regions according to the statistics of the fibers color. Concretely speaking, the arbitrary pixels $(i, j)$ in the fibers' regions must meet the following two conditions:

1) $0.3 \leq S(i, j) \leq 0.6$

2) $160 \leq H(i, j) \leq 240$ or $270 \leq H(i, j) \leq 360$

After the images are segmented, the value of the target pixel is denoted as 255 and that of the background pixel is 0 .

In order to avoid fibers' breaks at the point of rough location, a dilation operator of mathematical morphology is employed to filter the segmented images. The size of the filter window is $5 \times 5$.

(3) Fine segmentation of fibers

In the process of the coarse segmentation of components $\mathrm{H}$ and $\mathrm{S}$, the threshold setting is slack. 
Although the fibers pixels will not be missed in this way, some non-cellulose pixels will be mistakenly included in the detection. So, a fine segmentation of the fibers regions roughly located is proposed. The statistics shows that although the fibers pixels are not so bright in the whole image their brightness is significant when they are compared to their nearby background pixels in the fibers neighboring regions. So for the component V, this article suggests adopting more stable and adaptive threshold segmentation method called OTSU to carry out a fine division of the images and remove some mistakenly detected non-cellulose pixels in the fibers regions acquired through the coarse segmentation. The method to obtain the $\mathrm{V}$ component images in the fibers regions is: first, to calculate the largest external rectangular region of every fiber in the images roughly segmented; then, to capture the corresponding grayscale image of this rectangular region. And the method to get the threshold value of OTSU is as follows.

Computes the appearance probability of the gray value $i$ of the image: $p(i)=n_{i} / N$ ( $i=0,1, \ldots, L-1)$. $\mathrm{N}$ is the total number of the image pixels. $n_{i}$ is the number of the pixels whose gray value is $i . L$ is the image's gray level. The whole image can be divided into two parts categorized as target $(M)$ and background $(B)$ by using the threshold value $x$. Suppose that the gray value of the target is less than that of the background. Let

$$
\begin{aligned}
& w_{M}(x)=\sum_{i=0}^{x} n_{i} / N, w_{B}(x)=\sum_{i=x+1}^{L-1} n_{i} / N, \\
& \mu_{M}(x)=\left(\sum_{i=0}^{x} i \cdot p(i)\right) / w_{M}(x), \mu_{B}(x)=\left(\sum_{i=x+1}^{L-1} i \cdot p(i)\right) / w_{B}(x),
\end{aligned}
$$

then the between-class variance is

$$
\sigma(x)=w_{B}(x) w_{M}(x)\left(\mu_{B}(x)-\mu_{M}(x)\right)^{2}
$$

Selects the best threshold $x^{*}$ from $[0, L-1]$ which has the maximum between-class variance,

$$
x^{*}=\operatorname{Arg}\left\{\max _{0 \leq x \leq L-1} \sigma(x)\right\}
$$

The pixels whose gray value is greater than $x^{*}$ can be regarded as those of the fibers targets (with gray values of 255) and the other pixels can be judged as those of the background (with gray value of 0 ).

\section{(4) Filtering fibers}

The median filter combined with mathematical morphology can be used to filter out noises and false fibers targets. The steps are as follows.

Step 1: uses the median filter to eliminate the isolated noise points. The size of the filter window is $3 \times 3$.

Step 2: uses the open operator of mathematical morphology to further filter out noises and to fill up holes. The size of the filter window is $3 \times 3$.

Step 3: uses 8-adjacency connecting method to mark the detected binary images. In addition, because the number of the pixels contained in the fibers targets is in a limited range (according to the statistics: 40 200. The unit: pixel), the dual threshold method can be used to remove the false targets. The lower limit of the threshold is 40 and its upper limit 200.

\section{Experiment simulation and analysis}

In order to prove that the proposed method is effective in bill fibers extraction, its performance is evaluated from both quantitative and qualitative perspectives.

(1) Qualitative evaluation

Fig. 2 shows the images' segmentation result of the proposed method. The bill input image is shown by Fig.1 (a). Fig.2 (a) is the result of the roughly located fibers and Fig.2 (b) is the fibers' final segmentation result. Subjectively speaking, there are many false targets fibers in the stage of 
rough location while after the fine division those targets are filtered out. Therefore, the segmentation result is in accord with the fibers' real distribution.
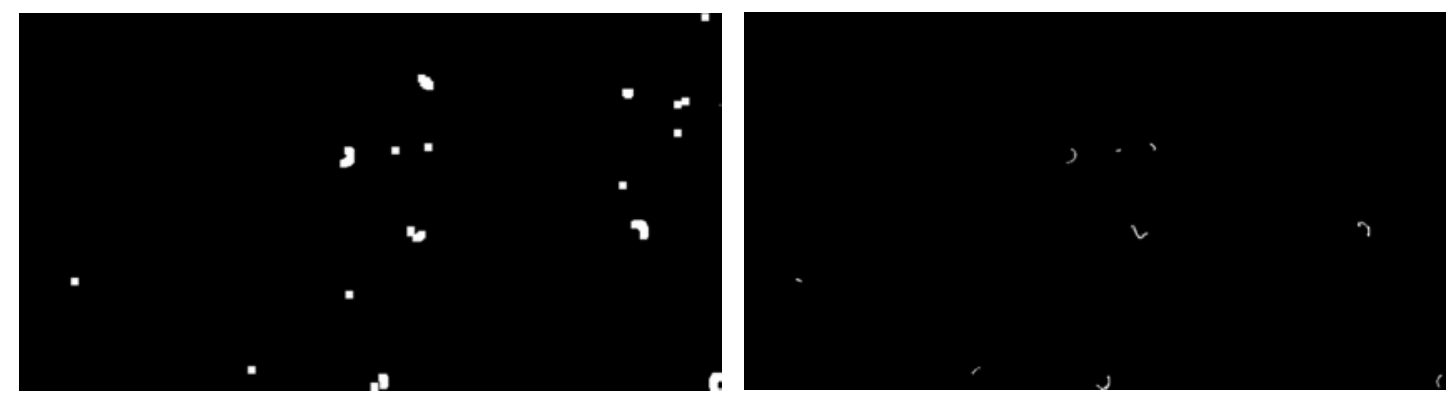

Fig.2 The segmentation result of our method

Fig.3 shows the same input images' segmentation results of the presently mainstream segmentation methods (the segmented images are filtered by using the filtering method mentioned in 2.4 to remove such interferences as mission flowers and theme patterns). Fig.3 (a) and Fig.3 (b) are the segmentation result of the methods stated in literature [5] and [6], respectively. Generally speaking, compared to these two methods, the proposed method has a better segmentation result and the fibers targets extracted by using it more conform to their real distribution as is shown in Fig. 1 (a). Accordingly, its extraction effect is apparently better than the other two.

Fig.3 The segmentation results of mainstream methods

(2) Quantitative evaluation

This paper chooses the fibers quantity deviation $N$ and the fibers matching ratio $M$ as the criteria of the quantitative evaluation. Suppose the number of a bill's fibers is manually marked as $N 1$ and that the number automatically detected is $N 2$. The number of the fibers which have matching characters is supposed to be $N 0$ (here matching means that the relative location of the center of gravity of two fibers is less than 3 pixels), then

$$
\begin{aligned}
& N=|N 1-N 2| \\
& M=\frac{2 \times N 0}{N 1+N 2} \times 100 \%
\end{aligned}
$$

Table 1 shows the contrast of the performance index of different methods. The experiments choose altogether 500 bill images and two indexes are the mean value of the result of the treated images. It is obvious that the proposed method has lower quantity deviation and higher fibers matching ratio. The main reason is that this method can overcome the interference from the complicated background of a bill and is more adaptive in the segmentation stage. In addition, this method is fast in processing and only needs less than 0.2 second on average to segment a bill image with the area of $640 \times 364$ (computer platform: CPU 3.20 GHz quad core, memory 4GB), so it can satisfy the speed need in bill authenticity identification. 
Table 1 Performance comparison

\begin{tabular}{|c|c|c|c|}
\hline $\begin{array}{c}\text { Method } \\
\text { s }\end{array}$ & $\begin{array}{c}\text { literature } \\
{[5]}\end{array}$ & $\begin{array}{c}\text { literature } \\
{[6]}\end{array}$ & $\begin{array}{c}\text { our } \\
\text { method }\end{array}$ \\
\hline $\mathrm{N}$ & 5.1 & 3.6 & 1.2 \\
\hline $\mathrm{M}$ & $83.4 \%$ & $89.9 \%$ & $97.8 \%$ \\
\hline
\end{tabular}

\section{Conclusions}

In order to solve the problem of high classifying error in bill fibers extraction, an improved method is proposed based on global statistic decision and local adaptive segmentation. According to this method, first, in the $\mathrm{H}, \mathrm{S}$ color space, a primary mapping is used to roughly locate the suspicious fibers regions. Then, in the $\mathrm{V}$ space of the regions, a more adaptive threshold segmentation method called OTSU is adopted to finely divide the images. Finally, mathematical morphology is employed to filter the fibers and at the same time reduces classifying error. The experiments show the proposed method is reliable in the segmentation of the fibers targets.

\section{References}

[1] Xie J, Liu T, Chen Z, et al. Joint template matching algorithm for associated multi-object detection.[J]. Ksii Transactions on Internet \& Information Systems, 2012, 6(1):393-403.

[2] Ohtsu N. A Threshold Selection Method from Gray-Level Histograms[J]. Systems Man \& Cybernetics IEEE Transactions on, 1979, 9(1):62-66.

[3] Kapur J N, Sahoo P K, Wong A. A new method for gray-level picture thresholding using the entropy of the histogram[J]. Computer Vision Graphics Image Processing, 1985, 29(3):273-285.0

[4] Madhukumar S, Santhiyakumari N. Evaluation of k-Means and fuzzy C-means segmentation on MR images of brain[J]. Egyptian Journal of Radiology \& Nuclear Medicine, 2015, $1(2): 475-479$.

[5] Ren Z, Shakhnarovich G. Image Segmentation by Cascaded Region Agglomeration[C]// IEEE Conference on Computer Vision \& Pattern Recognition. 2013:2011-2018.

[6] An N Y, Pun C M. Color image segmentation using adaptive color quantization and multiresolution texture characterization[J]. Signal Image \& Video Processing, 2014, 8(5):943-954.

[7] Erhu Z, Fan W, Yongchao L, et al. Automatic detection of microcalcifications using mathematical morphology and a support vector machine.[J]. Bio-medical materials and engineering, 2014, 24(1):53-59. 